\title{
Beneath the sword of Damocles: regenerative medicine and the shadow of immunogenicity
}

Few topics in regenerative medicine have inspired such impassioned debate as the immunogenicity of cell types and tissues differentiated from pluripotent stem cells. While early predictions suggested that tissues derived from allogeneic sources may evade immune surveillance altogether, the pendulum has since swung to the opposite extreme, with reports that the ectopic expression of a few developmental antigens may prompt rejection, even of tissues differentiated from autologous cell lines. Here we review the evidence on which these contradictory claims are based in order to reach an objective assessment of the likely magnitude of the immunological challenges ahead. Furthermore, we discuss how the inherent properties of pluripotent stem cells may inform strategies for reducing the impact of immunogenicity on the future ambitions of regenerative medicine.

First draft submitted: 20 September 2016; Accepted for publication: 11 October 2016; Published online: 1 December 2016

Keywords: allograft rejection $\bullet$ embryonic stem cells $\bullet$ haplobank $\bullet$ immune suppression - induced pluripotent stem cells $\bullet$ regulatory T cell $\bullet$ transplantation tolerance

The first derivation of embryonic stem cell lines (ESC) from supernumerary human blastocysts [1] provided more than our first glimpse into the earliest stages of human development or a novel platform for drug discovery. The prospect of using lines of human ESC (hESC) as an inexhaustible supply of cell types and tissues for cell replacement therapy (CRT) heralded the present era of regenerative medicine. The promise of novel treatments to address the rising incidence of chronic and degenerative diseases was fueled by reports of the differentiation of therapeutically relevant cell types as diverse as retinal pigmented epithelium (RPE) [2], dopaminergic neurons [3] and insulin-responsive $\beta$-like cells of the pancreatic islets [4,5]. Only recently have these early studies translated into Phase I/II clinical trials for the treatment of acute spinal cord injury and diseases of the eye, such as age-related macular degeneration and Stargardt's disease, for which early outcomes have already been reported [6,7]. Furthermore, a number of trials are either planned or currently underway for the treatment of heart failure, Parkinson's disease and Type I diabetes, for which appropriate adult stem cells are difficult to access and expand ex vivo, making pluripotent stem cells (PSC) an attractive alternative source [8].

Nevertheless, the first clinical application of PSC has brought into sharp focus the need for engraftment, functional integration and long-term persistence of administered cells, making an encounter with the immune system of the recipient inevitable and raising the spectre of immunological rejection that continues to confound solid organ transplantation for the treatment of end-stage organ failure. While the demonstration of induced pluripotency in 2006 [9] was anticipated to overcome many of the immunological obstacles to clinical translation, the advent of induced PSC (iPSC) has, in reality, served
Paul J Fairchild ${ }^{*, 1}$, Christopher Horton', Priyoshi Lahiri', Kumaran Shanmugarajah' \& Timothy J Davies'

'Sir William Dunn School of Pathology, University of Oxford, South Parks Road, Oxford, OX1 3RE, UK

*Author for correspondence: Paul.Fairchild@path.ox.ac.uk

Future
Medicine $\mathrm{fS}$ 
merely to introduce further uncertainty and heated debate into a field already well acquainted with controversy. Given that almost 20 years have now passed since the first description of hESC and 10 years since the seminal demonstration of induced pluripotency, it is perhaps timely to ask how much we truly know about the nature and magnitude of the immunological challenges that lie ahead and how far we have come in developing an effective strategy to address them.

\section{The vexed question of immunogenicity}

Early discussion of the therapeutic potential of hESC invited speculation as to how the immune system of potential recipients might perceive tissues differentiated from them, some laboratories predicting that they may ultimately prove to be immunologically silent. This premise drew on various sources of evidence, not least of which was the recognition that replacement tissues would most likely be devoid of endogenous dendritic cells (DCs) (Figure 1). These specialized antigenpresenting cells are normally carried over from the donor as stowaways within the parenchyma of organ allografts, their subsequent migration to the secondary lymphoid tissues of the recipient facilitating the direct presentation of products of the MHC to naive alloreactive $\mathrm{T}$ cells, catalyzing their polyclonal activation and acute rejection of the graft (Box 1) [10]. However, it was argued that not only might replacement tissues differentiated in vitro lack DC in the absence of the appropriate growth factors and environmental cues, but that such 'designer tissues' would also lack the lymphatic drainage required for the emigration of any rogue DC that had arisen spontaneously, rendering ineffective a critical route of allosensitization.

Equally fundamental were early observations that ESC are profoundly deficient in expression of polymorphic MHC molecules, both class I and II [14], a likely vestige of their unconventional origin from early blastocysts whose principal agenda is to evade allorecognition by the maternal immune system. The lack of expression of MHC determinants and absence of costimulatory molecules, such as CD80 and CD86, were shown to correlate with the inability of undifferentiated ESC to stimulate allogeneic $T$ cells in vitro [15] while failing to provide a source of alloantigen that could be reprocessed by recipient DC for presentation in a selfMHC-restricted manner (Box 1). While this indirect route of allosensitization has long been appreciated to elicit the chronic rejection of solid organ allografts, a form of rejection notoriously resistant to immune suppression, its compromise in the context of CRT suggested further grounds for optimism. Furthermore, in the absence of endogenous MHC expression by the grafted tissues, host DC circulating through the graft would fail to acquire on their surface whole allogeneic MHC determinants by the process of trogocytosis, which might otherwise have activated recipient $\mathrm{T}$ cells via the so-called semi-direct pathway (Box 1).

In addition to the passive inability of PSC to elicit responses among alloreactive $\mathrm{T}$ cells, various studies highlighted their capacity to actively exert nonspecific suppressive effects on $\mathrm{T}$ cells stimulated within the local vicinity. Such bystander effects have been variously attributed to the secretion of prostaglandin $\mathrm{E}_{2}$ [16] and the expression of hemoxygenase-1 [17], known to have anti-inflammatory effects via the release of carbon monoxide through the catabolism of heme. Furthermore, ESC have been implicated in the consumption of essential amino acids from the local microenvironment, thereby depriving $T$ cells of the critical nutrients on which they rely for their activation and clonal expansion. For example, Yachimovich-Cohen et al. demonstrated the constitutive expression of arginase-1 by both mouse and human ESC, and the resulting depletion of local arginine. The resulting suboptimal environment was shown to induce the downregulation of $\mathrm{CD} 3 \zeta$ by local $\mathrm{T}$ cells and inhibition of their cytokine secretion and proliferation, an effect abolished by the treatment of hESC with a specific arginase-1 inhibitor [18].

While mouse and human iPSC have been shown to share many of the immunosuppressive properties of ESC in vitro [19], the epigenetic memory they display for the cell type of origin [20,21] provides additional leverage for intervening in any immune response elicited upon their downstream administration to recipients. Wang and colleagues demonstrated the feasibility of deriving iPSC lines from Sertoli cells of the mouse testis, an anatomical site endowed with immune-privileged properties [22]: by enshrining in the resulting iPSC, the epigenetic signature associated with immune privilege, the resulting cells were found to be significantly less immunogenic when transplanted to allogeneic recipients than conventional iPSC derived from dermal fibroblasts [23]. Similar findings were reported in vitro for neural progenitors derived from iPSC reprogrammed from umbilical cord mesenchymal cells, the equivalent population derived from conventional iPSC eliciting significantly greater proliferation and cytotoxic $\mathrm{T}$ lymphocyte (CTL) activation in co-cultures [24]. Thus, in addition to intrinsic features of PSC that are naturally immune suppressive, the gene expression profile associated with mechanisms of immune privilege may also be exploited to reinforce the capacity of iPSC to evade immune recognition. Paradoxically, however, the very same studies showed progressive increase in immunogenicity of cell types differentiated from iPSC with ongoing passage of the parent cell line, known 
Box 1. Mechanisms of allograft rejection.

Over the last few decades, research into solid organ transplantation has revealed a number of distinct mechanisms that may conspire to induce allograft rejection [10]. 'Hyperacute' rejection occurs within minutes or hours of implantation and is caused by naturally occurring antibodies specific for carbohydrate residues expressed by endothelial cells of the implanted organ. Antibody binding results in complement activation and widespread vascular damage. Such an aggressive form of rejection typically occurs in the context of xenotransplantation or in response to a mismatch of ABO blood group antigens between donor and recipient and is, therefore, comparatively straightforward to avoid. In contrast, acute rejection is catalyzed by dendritic cells (DCs) carried over from the donor within the parenchyma of the organ. In response to the inflammatory insults caused by surgery, DCs undergo a process of maturation and migrate to the secondary lymphoid tissues of the host. Here, they present whole allogeneic MHC molecules to the T-cell repertoire, inducing a diverse polyclonal response among naive T cells, estimates suggesting that between 5 and $10 \%$ of all T cells may respond. The recognition of intact foreign MHC molecules by T cells is known as the 'direct pathway' of alloantigen presentation [11]. The so-called 'indirect' pathway involves the acquisition, by host DCs, of foreign MHC molecules and any other minor histocompatibility antigens by which donor and recipient may vary, and their processing and presentation to alloreactive T cells as peptide fragments bound to the recipient's own MHC determinants [11]. Given that the precursor frequency of T cells responding to such peptide-MHC complexes is no greater than for conventional foreign antigens, the resulting T-cell response is less dramatic, but leads, instead, to a chronic form of rejection, sometimes triggered by concomitant infection, which is less amenable than acute rejection to intervention by immune suppression [12,13]. Interestingly, a hybrid of these two routes of sensitization has also been described, known as the 'semi-direct' pathway which relies on immigration into the grafted tissues of recipient DCs. These not only acquire alloantigens from dead and dying cells that are subsequently processed for presentation via the indirect route, but also acquire intact allogeneic $\mathrm{MHC}$ molecules from neighbouring cells by stealing small portions of the plasma membrane through a process known as 'trogocytosis'. These DCs are, therefore, uniquely capable of simultaneously presenting alloantigens via both direct and indirect pathways.

to be responsible for erasing the epigenetic memory of the cell type of origin [21], an epigenetic memory that may also be lost upon directed differentiation in vitro. These findings raise the important question of whether the properties of PSC per se are relevant to issues of immunogenicity, given that it is the tissues differentiated from them to which the immune system of the recipient will ultimately be exposed.

To address these issues directly, Drukker et al. administered hESC or their differentiated progeny under the kidney capsule of mice carrying peripheral blood mononuclear cells of human origin. While control grafts of adult tissues were promptly rejected, the authors detected minimal inflammatory infiltrates in grafts of hESC, irrespective of their extent of differentiation, a finding attributed, at least in part, to deficiencies in the direct pathway of alloantigen presentation (Figure 1) [25]. Although far-reaching for the emerging field of regenerative medicine, these findings were not supported by subsequent studies in which an inoculum of ESC, administered directly to the heart of allogeneic recipients, showed escalating immunogenicity that correlated with the differentiation status of the cells [26]. Perhaps more surprising, however, were findings that undifferentiated allogeneic ESC were likewise ultimately rejected despite their paucity of MHC expression, the absence of accompanying DC and a raft of immunosuppressive mechanisms to derail T-cell activation $[27,28]$, such in vivo data calling into question the very premise on which early assumptions of low immunogenicity were founded [29,30].
In an attempt to define more rigorously the level of immunological disparity that might be tolerated in the context of CRT, our own laboratory derived a panel of mouse ESC that differed from recipient mice at defined genetic loci representing an escalating level of immunological disparity and implanted tissues differentiated from them under the kidney capsule in the absence of immune suppression in order to assess the magnitude of the subsequent immune response. Surprisingly, even tissues genetically identical to the recipient strain at every locus with the exception of male-specific antigen and a single MHC class I gene, were robustly rejected, albeit at a slightly slower tempo than tissues differentiated from fully allogeneic ESC [31]. These findings suggested that despite numerous properties anticipated to render ESC and their progeny immunologically privileged, the immune response of the recipient remains a formidable opponent, which we ignore at our peril.

\section{Production of a stem-cell 'haplobank': a solution to immunogenicity?}

Mounting evidence that PSC and their derivatives are likely to be subject to the very same immune response that has traditionally confounded whole organ transplantation has inspired numerous calls over the past decade for the establishment of an international bank of cGMP-compliant cell lines in order to facilitate rudimentary matching with potential recipients [32-34]. Such ambitious plans received significant support from early estimates of the number of hESC lines that might be required to ensure a beneficial match for an equi- 


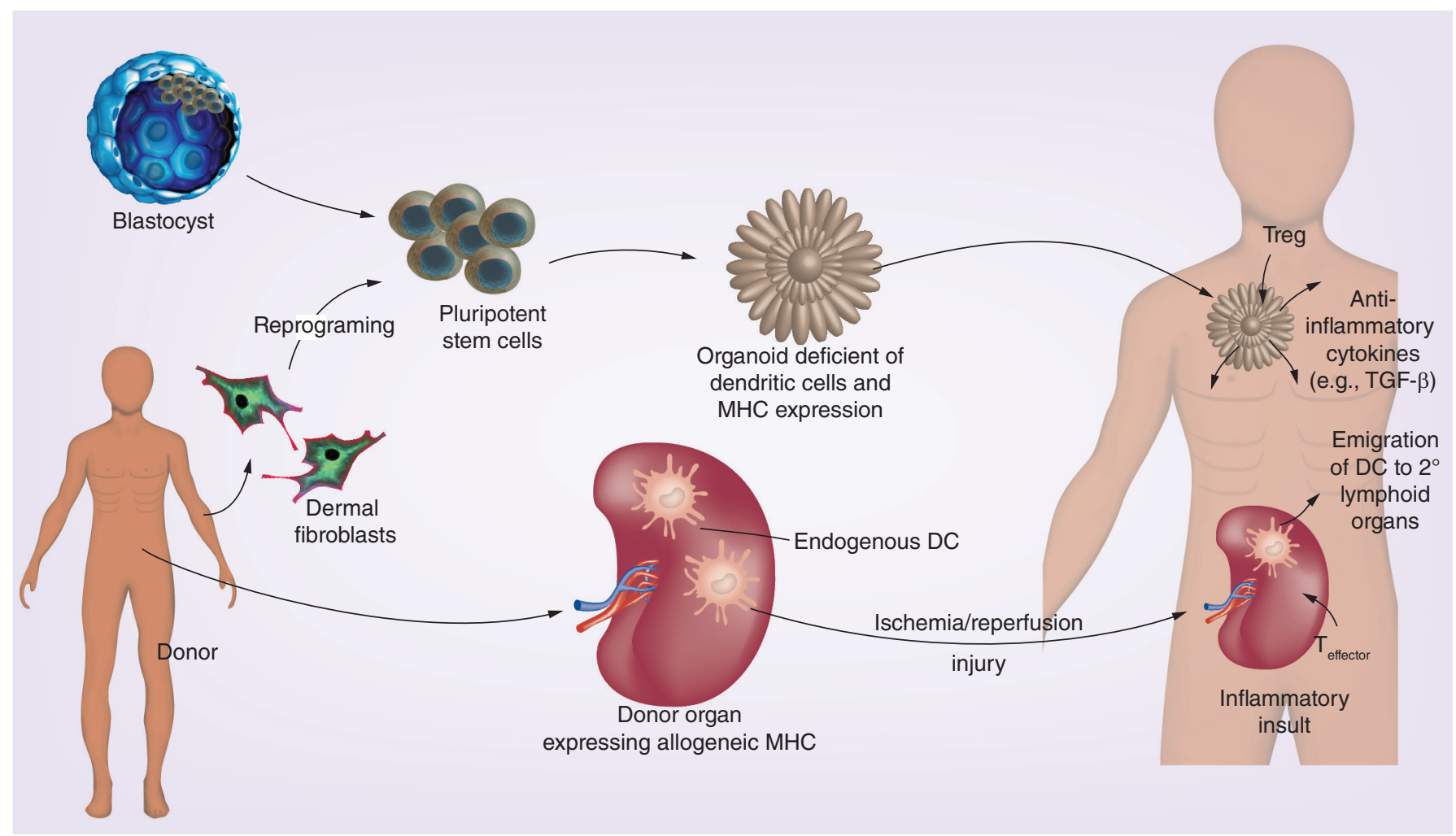

Figure 1. Comparison of the immunological challenges of cell replacement therapy and solid organ transplantation. Unlike solid organs from living or cadaveric donors, tissues or organoids differentiated from PSC lack endogenous DC capable of migrating to the secondary lymphoid tissues of the recipient and eliciting acute rejection of the graft. Furthermore, replacement tissues express constitutively low levels of MHC molecules but secrete anti-inflammatory cytokines such as TGF- $\beta$, creating a microenvironment conducive to the polarization of infiltrating $T$ cells toward a regulatory phenotype following implantation. Conversely, solid organ transplantation is associated with ischemia/reperfusion injury that triggers a localized inflammatory insult, further sustaining the activation of effector $T$ cells entering the graft.

DC: Dendritic cell; MHC: Major histocompatibility complex; PSC: Pluripotent stem cell.

table proportion of the UK [35] or Chinese [36] populations. Despite over 9000 alleles having so far been identified within the HLAs, the human equivalent of the MHC, as few as $150 \mathrm{hESC}$ lines were estimated to provide a full match at HLA-A, -B and -DR loci for up to $20 \%$ of the UK population and a partial match for almost $85 \%$ [35]. Furthermore, given that there is significant linkage disequilibrium among genes within the HLA, Taylor estimated that as few as ten hESC lines, homozygous for common HLA types, would provide a complete match at these critical loci for almost 38\% of the UK population. Although subsequent analysis failed to identify any such homozygous 'superdonors' among existing hESC lines available in the USA, questioning the feasibility of achieving even such a modest goal [37], the subsequent advent of induced pluripotency has significantly changed the dynamics involved, making the construction of a 'haplobank' of iPSC a far more credible proposition than for their embryonic counterparts [38,39].

While the generation of hESC lines is wholly dependent on the availability of blastocysts surplus to requirements in in vitro fertilization (IVF) clinics, for which the HLA haplotype is unknown at the time of derivation, it would doubtless prove feasible to actively recruit iPSC donors with a desirable haplotype currently missing from the bank, as well as members of ethnic minorities that are less likely to frequent IVF clinics. The resulting bank of cGMP-compliant iPSC lines may, therefore, be far more inclusive and representative of a racially and ancestrally diverse population than could ever be achieved with hESC [32]. Most importantly, however, iPSC lines could be 'commissioned' from superdonors homozygous across the MHC, many of whom are already identifiable from databases of bone marrow or umbilical cord blood donors [40], such recruitment initiatives posing few ethical constraints beyond the need for informed consent, compared to those surrounding the use of supernumerary human embryos.

Although the availability of an international haplobank of iPSC would undoubtedly help reduce the immunological challenge of CRT, there is growing consensus that such a strategy is unlikely to fully dis- 
pel the threat of rejection in the absence of immune suppression. For instance, the practice of matching at only selected HLA loci is based on decades of research into solid organ transplantation, which has revealed identity at HLA-A, -B and -DR to confer the greatest benefit on outcome, the inclusion of additional loci providing only marginal further benefit while serving to drastically decrease the likelihood of finding an appropriate match. However, such laxity is unlikely to succeed in the context of CRT in which recourse to long-term immune suppression may not be an option, given the recognized risks of tumorigenesis. In particular, gene products encoded within the HLA-C, -DP and -DQ loci remain highly immunogenic: indeed, studies in the mouse showed the rejection of tissues differentiated from male ESC by female recipients disparate at just a single MHC class I gene [31]. Importantly, these same studies revealed an even more insidious threat to the integrity of grafts derived from PSC: by deriving ESC lines from a strain of mouse identical to the recipient across the entire MHC yet disparate at so-called 'minor' histocompatibility $(\mathrm{mH})$ antigens, we found robust rejection of tissues differentiated from them over a time frame indistinguishable from fully allogeneic grafts [31]. While in the mouse, $\mathrm{mH}$ antigens are known to consist of mitochondrial proteins and the products of naturally occurring polymorphic genes, their identity in the human is far from certain, making any attempt to match at these additional loci ill-founded on both scientific and pragmatic grounds. Consequently, there is little doubt that, although clearly beneficial, the construction of an iPSC haplobank will require additional strategies to deal with residual immunogenicity. While some have advocated encapsulation of tissues to provide a protective barrier against the recipient's immune system, numerous recent studies have uncovered more subtle opportunities for immune intervention.

\section{Prospects for the induction of transplantation tolerance}

While the burden of evidence currently suggests that PSC and their progeny succumb to immune surveillance in much the same way as transplanted tissues from conventional sources, several studies have uncovered a fragile form of immune privilege rendered visible under contrived experimental conditions [31,41]. Our own studies made use of mice expressing a transgenic T-cell receptor specific for a defined MHC class II-restricted epitope from the male-specific $\mathrm{mH}$ antigen, Dby, which confers on female mice the capacity to robustly reject male tissues [42]. Surprisingly, however, tissues differentiated from karyotypically-male ESC survived indefinitely when implanted under the kidney capsule of female T-cell receptor transgenic recipients, despite attracting a significant $\mathrm{CD}^{+}{ }^{+}$ T-cell infiltrate [31]. This paradox was resolved by gene expression profiling, which revealed the constitutive expression within the graft of TGF- $\beta$, responsible for polarizing infiltrating $\mathrm{T}$ cells toward a regulatory phenotype, so-called induced Treg (iTreg) (Box 2). Indeed, in mice carrying a dominant negative TGF- $\beta$ receptor (TGF $\beta R I I)$ within the T-cell compartment, none of the infiltrating cells had become polarized toward the Treg lineage, defined by their expression of the transcription factor FoxP3 [31]. In a similar vein, de Almeida et al. reported the secretion of TGF- $\beta$ by iPSC-derived endothelial cells, which contributed to their own survival following implantation through recruitment of Treg cells. Infiltrating Treg were found to be responsible for secretion of the anti-inflammatory cytokine IL-10 within the local microenvironment of accepted grafts, the neutralization of which prompted their subsequent rejection [43]. Likewise, Sugita et al. showed constitutive expression of TGF- $\beta$ by RPE differentiated from human iPSC, which supported polarization of responding $\mathrm{T}$ cells toward a $\mathrm{CD} 25^{+} \mathrm{FoxP} 3^{+}$ phenotype in vitro [19]. Collectively, these findings suggest that the weak propensity for immune privilege displayed by PSC, although normally inadequate to prevent rejection across any barrier greater than that posed by $\mathrm{mH}$ differences, might nevertheless be harnessed therapeutically to secure dominant transplantation tolerance across far greater immunological disparities (Figure 1).

Such predictions have been borne out by experiments using complementary approaches. First, Rong et al. genetically modified hESC to express two immunomodulatory molecules, CTLA4Ig, capable of antagonizing co-stimulatory pathways, and the inhibitory receptor PD-L1. This combination was found to secure indefinite survival of tissues following their transplantation into humanized NSG mice, either molecule alone proving insufficient [46]. Given that prior genetic modification of PSC may pose significant obstacles to downstream translation, a number of groups have sought to replicate the state of tolerance achieved through administration of various combinations of monoclonal antibodies (mAb) and soluble agents capable of targeting co-receptor or co-stimulatory pathways. For example, the administration of a short course of nondepleting $\mathrm{mAb}$ specific for mouse CD4 and CD8, was found to permit the engraftment of tissues differentiated from fully allogeneic ESC [31], while the introduction of anti-CD40L into the conditioning regime, secured survival of xenogeneic tissues from hESC by outbred CD1 recipient mice [47]. Similar outcomes were reported by Pearl et al. using the 


\section{Box 2. Treg and transplantation tolerance.}

The existence of Treg was first formally demonstrated in seminal studies of transplantation tolerance, which illustrated their capacity to pass on the tolerant state to naive T cells by encouraging their commitment to the same Treg lineage, a process referred to as 'infectious tolerance' [44]. There are now known to be two populations of Treg, natural Treg that are selected centrally within the thymus, and induced Treg (iTreg) that have adopted a regulatory phenotype in the periphery due to the context and local microenvironment in which they first recognized antigen. Both subsets of Treg are CD4+ MHC class II-restricted cells that constitutively express CD25 and the transcription factor FoxP3, the loss of which in humans is associated with IPEX syndrome, a wasting disease characterized by multiple autoimmune responses. It is the loss of activity of natural Treg that is largely responsible for the associated pathology since their role is primarily to maintain tolerance to self-antigens. In contrast, iTreg are better adapted to preventing unwanted immune responses to harmless foreign antigens, such as components of the gut microflora. Indeed, phylogenetically, the first appearance of iTreg in eutherian mammals, strongly suggests that they first evolved to establish a temporary state of tolerance to paternal alloantigens expressed by the developing fetus [45]. It is this physiological role that has been exploited in protocols for the induction of transplantation tolerance that convert alloreactive T cells to protective iTreg by depriving them of the essential co-receptor and co-stimulatory signals required for their normal activation and the acquisition of effector function.

combination of soluble CTLA4Ig together with $\mathrm{mAb}$ specific for CD40L and LFA-1: indeed, a short course of treatment with these agents around the time of grafting proved significantly more effective at securing long-term graft survival than conventional immune suppression [48].

Although not all demonstrations of transplantation tolerance in the context of CRT have found a role for Treg cells [49], the majority have identified $\mathrm{CD} 25^{+} \mathrm{FoxP}^{+}$ cells to be fundamental to the maintenance of the tolerant state [47]. For instance, Grinnemo et al. demonstrated the accumulation of Treg around tolerated grafts of hESC delivered to the testis of immune competent mice under the cover of co-stimulatory blockade [50], while similar results were obtained when hESC were injected under the kidney capsule, an anatomical site not considered to enjoy immune-privileged status [51]. That Treg actively participate in the maintenance of tolerance was demonstrated by their ablation with $\mathrm{mAb}$ specific for CD25, which routinely precipitated graft rejection [52]. Furthermore, in an attempt to develop a protocol for tolerance induction more amenable to clinical implementation, Pan et al. used total lymphoid irradiation of mice in combination with the adoptive transfer of Treg to achieve indefinite survival. Importantly, Treg derived from the recipient proved equally effective to cells of donor origin, greatly simplifying downstream clinical protocols [53].

Collectively, these findings bode well for the future of regenerative medicine, suggesting that the intrinsic properties of tissues derived from PSC resonate with the mechanisms of dominant transplantation tolerance, enabling them to participate fully in their own survival. Consequently, it would appear to be significantly easier to achieve the indefinite survival of replacement tissues differentiated from a pluripotent source than equivalent cell types and tissues from conventional donors. While such a conclusion is clearly to be welcomed, the advent of iPSC that may, in prin- ciple, be derived from individuals in a fully autologous manner, has questioned whether tolerance induction is actually necessary to realize the vision of regenerative medicine.

\section{Tales of the unexpected: could autologous tissues ever be rejected?}

The seminal demonstration that somatic cells of human origin could be reprogrammed to a state of pluripotency [54,55], paved the way for a personalized approach to regenerative medicine, free of the ethical sensitivities that had confounded the derivation of hESC: if only the health economics could be addressed, the technology was now available to enable the design of therapies, tailored to the personal requirements of each individual. Indeed, last year such a vision was finally realized following the ground-breaking treatment of a Japanese woman with age-related macular degeneration using RPE differentiated from autologous iPSC [56]. However, the unquestioned assumption that autologous cells would not be rejected by recipients, the very premise on which such trials were founded, was recently called into question. Zhao et al. derived iPSC lines from mouse embryonic fibroblasts and administered them subcutaneously to genetically identical or 'syngeneic' recipients in order to simulate the autologous setting. Surprisingly, while syngeneic ESC formed teratomas as anticipated, iPSC with the very same genetic background attracted a significant T-cell infiltrate that led to their rejection [57]. Similar findings were subsequently reported by de Almeida et al., although the extent of immunogenicity appeared to decrease upon subsequent differentiation [43]. In order to investigate whether similar issues might be encountered following implantation of cells of human origin, Zhao et al. generated mice harboring a human immune system and derived human iPSC (hiPSC) from the same human fetal liver used to reconstitute the mice. The subcutaneous administration of undifferentiated iPSC led 
to the formation of teratomas that likewise attracted a significant inflammatory infiltrate, which ultimately compromised their survival [58]. Furthermore, some terminally differentiated cell types, such as smooth muscle cells, retained significant immunogenicity that led to their demise upon transplantation.

Given the adverse impact, such findings have had on the regenerative medicine sector, to say nothing of the uncertainty and additional risk that has had to be incorporated into nascent business plans, the underlying mechanisms involved have necessarily taken center stage and appear to revolve around the ectopic expression of certain genes by iPSC and their differentiated progeny that encode what might best be described as 'developmental antigens'. Pluripotency is associated with a pattern of gene expression that is normally lost shortly after implantation, precluding any participation of the associated gene products in selection of the T-cell repertoire and the induction of immunological tolerance once thymus organogenesis has occurred later in ontogeny (Figure 2). Zhao and colleagues identified nine genes that fulfilled the criteria of being strongly upregulated upon reprogramming to pluripotency, but failing to be properly silenced upon subsequent differentiation in vitro. One such antigen, Hormad1, is normally restricted in adulthood to the highly immune-privileged environment of the testis [59] where it contributes to formation of synaptonemal complexes during meiosis [60]. That self-tolerance to Hormad1 is profoundly compromised, is evident from its identification as a putative tumor-associated antigen for both testicular [61] and ovarian cancer [62], due to its intrinsic immunogenicity. It is, however, possible that the same immunogenicity, which may be exploited for the immunological destruction of testicular tumors, might also elicit destruction of cell types differentiated from iPSC in vitro that maintain its persistent expression following implantation in vivo (Figure 2).

While the demonstrable lack of self-tolerance to developmental antigens re-expressed through reprogramming provides a rational explanation for the loss of grafts derived from autologous iPSC (Figure 2), various studies have challenged these conclusions. First, techniques such as reverse transcription-PCR and flow cytometry failed to demonstrate any difference in the expression of developmental antigens encoded by genes such as Hormad1 and Zg16 between ESC and iPSC of human origin, irrespective of their state of differentiation [63], making it difficult to explain why the immune system of humanized mice might perceive the two sources so differently [58]. Equally important, however, are studies by Araki et al. who failed to detect any immune response in syngeneic recipients to tissues transplanted from chimeric mice, generated by the aggregation of early embryos with mouse iPSC [64]. Using a similar rationale, Wang et al. generated entire mice from iPSC using tetraploid complementation and transplanted skin, pancreatic islets or heart grafts into syngeneic recipients, thereby representing tissues from each of the three embryonic germ layers. All tissues were spontaneously accepted and continued to function normally for at least 3 months without any overt signs of rejection [65], questioning the validity of the original claims of immunogenicity. Nevertheless, although such in vivo studies might appear to carry significant weight, the introduction of iPSC into early embryos prior to implantation into the uterus of surrogate mothers, necessarily exposes them to the microenvironment of the inner cell mass, which may be conducive to the proper regulation of gene expression during development, countering the tendency for ectopic expression of developmental antigens observed during differentiation in vitro (Figure 2). Indeed, this possibility was supported by the failure of the authors to detect either Hormad1 or Zg16 in any of the grafted tissues [65]. Arguably, therefore, these studies may tell us little about the immunological challenges encountered in a more clinically-relevant scenario in which tetraploid complementation and the formation of chimeras are not viable approaches to the production of autologous replacement tissues and directed differentiation in vitro is, therefore, favored. However, even if the relevance of these studies is in doubt, independent sources of evidence likewise question the acquisition of immunogenicity by tissues differentiated from autologous iPSC [66]. For instance, in one study, the implantation of such tissues under the kidney capsule of syngeneic mice, failed either to provoke an inflammatory infiltrate or compromise the integrity of the graft [67]. Complementary experiments in nonhuman primates showed survival for more than a year of iPSCderived RPE transplanted into the subretinal space of autologous recipients, while allogeneic sources were rapidly rejected [68]. Furthermore, studies of neural transplantation in cynomolgus monkeys showed that, whereas autologous cells provoked minimal immune response, allografts elicited microglial activation and lymphocytic infiltration [69]. Given the contradictory outcomes that have been reported since the original findings of Zhao and colleagues, there is currently little consensus as to the extent of the immunological challenges that may be encountered in an autologous setting. Nevertheless, even in the worst case scenario that ectopic expression of developmental antigens renders iPSC-derived tissues targets of a T-cell response, it is perhaps worth noting the ways in which the nature of the response differs from bona fide rejection, providing significant opportunities for immune intervention. 


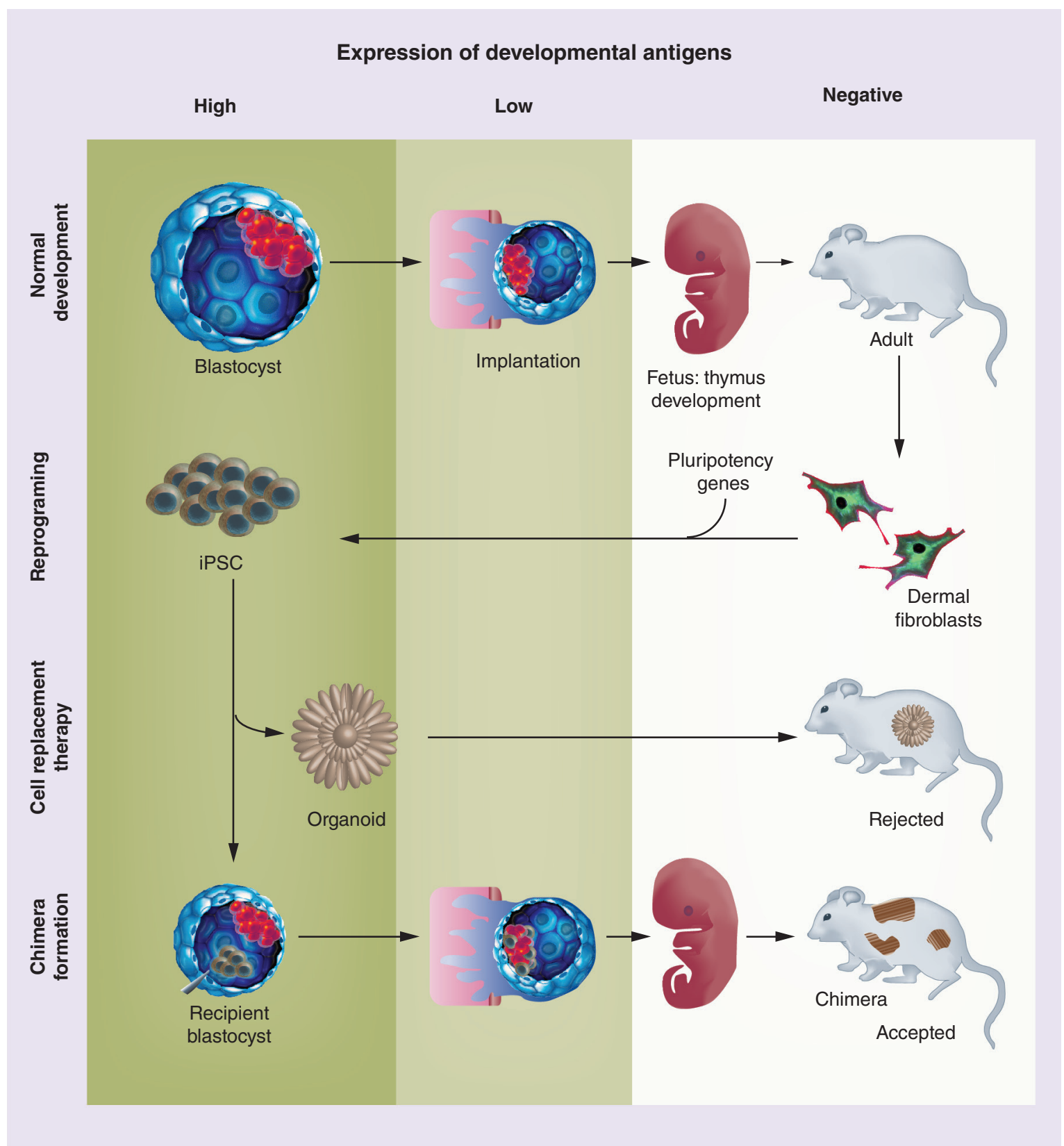

Figure 2. Immunogenicity of developmental antigens ectopically expressed by induced pluripotent stem cells and their differentiated progeny. Pluripotency-associated genes are expressed at high levels within the early embryo but are normally downregulated upon implantation, being extinguished long before thymic development and selection of the T-cell repertoire. Reprogramming of adult somatic cells to pluripotency is responsible for the re-expression of developmental antigens, which are not properly downregulated during differentiation in vitro, most likely accounting for their rejection, even by autologous recipients. Introduction of iPSC into the specialized environment of the early embryo may, however, restore the normal pattern of gene expression creating chimeras whose tissues are fully tolerated if transplanted to secondary recipients. iPSC: Induced pluripotent stem cell.

First, the response would be directed toward a limited repertoire of epitopes from developmental antigens presented in a self MHC-restricted manner, rather than products of the MHC per se. Consequently, there would be no role for conventional alloreactive $\mathrm{T}$ cells activated via the direct pathway of alloantigen presen- tation (Box 1). Such a scenario is, therefore, more closely allied to an autoimmune response than it is to allograft rejection; indeed one theory for the pathogenesis of myasthenia gravis is the re-expression in adulthood of the fetal- $\gamma$ chain of the nicotinic acetylcholine receptor, normally downregulated early during ontogeny and 
replaced for the rest of life by an alternative $\varepsilon$-chain. In the absence of tolerance to the $\gamma$-chain, however, its ectopic expression is thought to provoke an immune response, which subsequently expands to encompass additional self-antigens through the process of epitope spreading. Although the similarity with autoimmunity may not appear to provide grounds for optimism given the difficulty of re-establishing tolerance in an already primed immune system, CRT has the significant advantage of timing. Whereas an autoimmune response is well underway at the time of clinical presentation, and diversification of the T-cell repertoire is already advanced due to epitope spreading, tolerance may be established to a handful of predictable developmental antigens in anticipation of CRT before epitope spreading acts to diversify the nature of the T-cell response. Interestingly, those studies that have uncovered an immune response to autologous iPSC-derived tissues have shown the response to be at best oligoclonal, quite different in nature and magnitude from the T-cell repertoire infiltrating allogeneic grafts $[43,58]$. These findings suggest that such a response, however unwelcome, might be fully amenable to intervention, especially given the propensity for tolerance induction and the contribution of the tissue itself to the establishment and ongoing maintenance of the tolerant state.

\section{Conclusion \& future perspective}

The emerging field of regenerative medicine has reached an important milestone with the first-in-man use of autologous iPSC for the treatment of macular degeneration, the outcome of which may pave the way for similar intervention in numerous chronic and degenerative disease states in the future. Nevertheless, despite a decade of research into the immunological consequences of such an approach, many unknowns remain, introducing an unwelcome degree of uncertainty into planned and on-going clinical trials.

What is rather more certain, however, is that immune suppression, on which the success of whole organ transplantation continues to rely, is unlikely to provide the solution to immunogenicity in the context of CRT. At a scientific level, it has long been recognized that conventional immune suppression is effective at dealing with acute rejection, catalyzed by the direct pathway of alloantigen recognition, but is less successful at intervening in chronic rejection, fueled by the indirect pathway [12,13]. Given that tissues derived from PSC lack endogenous DC, we now know the indirect pathway to be primarily responsible for their recognition by the immune system of recipients, making chronic rejection the greatest threat to their indefinite survival and functional integrity. In addition, there are compelling ethical reasons for doubting the expediency of immune sup- pression, namely, that its long-term use may pose risks to patients that are substantially greater than the risks of some of the disease states, such as macular degeneration, amenable to treatment by CRT. Furthermore, the risk profile of such intervention is especially high in the light of the potential for malignant transformation of PSC [70,71]: to profoundly suppress the immune system of the recipient at the same time as implanting cells with a known propensity for tumorigenesis, would doubtless expose patients to an unacceptable level of risk. Arguably, therefore, the future success of the sector will be inextricably linked with the development of alternative strategies for immune intervention. While the challenge ahead remains considerable, there are, nevertheless, encouraging signs that the induction of immunological tolerance to PSC-derived tissues is not only feasible but significantly easier than the establishment of tolerance to tissues from conventional sources. In particular, the microenvironment created by PSCderived tissues uniquely resonates with the establishment and maintenance of dominant tolerance through the activity of Treg, suggesting that such grafts may actively participate in their own survival.

The legendary sense of foreboding that Damocles experienced, enshrined in roman mythology, offers a sobering perspective on the future of regenerative medicine. Damocles was permitted to survey all the wealth, privilege and power that accompany the office of king but with a sword suspended above his head by a single hair from a horse's tail, an attempt by King Dionysius, to convey just how precarious he felt his position to be. Like the sword of Damocles, immunogenicity threatens to derail the substantial riches and benefits to human health that regenerative medicine offers: only by developing effective strategies to modulate the immune response will the shadow cast by immunogenicity finally be dispelled.

\section{Acknowledgements}

The authors are grateful to S Hackett, P Sachamitr and A Leishman for helpful discussions.

\section{Financial \& competing interests disclosure}

Work on tolerance in the authors' laboratory is funded by the Regenerative Medicine Initiative of the Britain-Israel Research and Academic Exchange (BIRAX) Partnership and the UKRMP Immunomodulation Hub. PJ Fairchild serves on the Scientific Advisory Board of Universal Cells, Inc. The authors have no other relevant affiliations or financial involvement with any organization or entity with a financial interest in or financial conflict with the subject matter or materials discussed in the manuscript apart from those disclosed.

No writing assistance was utilized in the production of this manuscript. 


\section{Open access}

This work is licensed under the Creative Commons Attribution-NonCommercial 4.0 Unported License. To view a copy of this license, visit http://creativecommons.org/licenses/bync-nd/4.0/

Executive summary

Immunogenicity of tissues differentiated from pluripotent stem cells

- Early speculation that pluripotent stem cell (PSC) derived tissues may not be immunogenic was based on low expression of MHC determinants, the absence of endogenous dendritic cells and the immune suppressive properties of the parent cells.

- The weight of evidence now suggests that even tissues disparate at multiple minor histocompatibility antigens are recognized as foreign and succumb to rejection.

- Nevertheless, some experimental models have revealed a propensity for a fragile form of immune privilege that may be exploited for the induction of tolerance across major histocompatibility barriers.

Production of a stem cell halpobank

- Early calculations suggested that surprisingly few human embryonic stem cell lines might be required to make cell replacement therapy available to a significant proportion of an ethnically diverse population.

- The identification of donors homozygous within the HLA, the so-called 'superdonors', would further reduce the number of cell lines required.

- Induced pluripotency has made the construction of a halpobank far more feasible by allowing the recruitment of superdonors and other individuals with a desirable HLA haplotype.

- Although beneficial, evidence suggests that matching alone is unlikely to overcome the issues of rejection, necessitating alternative forms of immune intervention.

Induction of transplantation tolerance

- The constitutive secretion of TGF- $\beta$ by tissues differentiated from PSC creates an environment conducive to the polarization of infiltrating $T$ cells toward a Treg phenotype.

- The local microenvironment provided by the graft may be exploited for the induction of regulatory tolerance, either by forced expression of inhibitory receptors or administration of soluble agents capable of blocking co-receptor or co-stimulatory pathways.

- Induction of tolerance may prove easier to achieve to tissues derived from PSC than to equivalent tissues from conventional sources.

Immunogenicity of tissues derived from autologous-induced PSC

- Unexpected immunogenicity of tissues differentiated from autologous induced PSC has been attributed to the ectopic expression of 'developmental antigens', to which self-tolerance has not previously been established.

- Since various studies have failed to demonstrate immunogenicity in an autologous setting, the magnitude of the problem remains uncertain.

- Differences between the response to developmental antigens compared with allogeneic MHC determinants, suggest any immune response may more closely resemble autoimmunity than allograft rejection, suggesting possible strategies for immune intervention.

Future perspective

- Reliance on immune suppression is unlikely to provide a tenable solution to the issues of immunogenicity facing the regenerative medicine sector.

- By exploiting protocols for co-receptor or co-stimulation blockade that resonate with the properties of the microenvironment afforded by PSC-derived tissues, the induction of regulatory tolerance may be achievable in the future.

\section{References}

Papers of special note have been highlighted as:

- of interest; $\bullet \bullet$ of considerable interest

1 Thomson JA, Itskovitz-Eldor J, Shapiro SS et al. Embryonic stem cell lines derived from human blastocysts. Science 282, 1145-1147 (1998).

2 Idelson M, Alper R, Obolensky A et al. Directed differentiation of human embryonic stem cells into functional retinal pigment epithelium cells. Cell Stem Cell 5(4), 396-408 (2009).

3 Swistowski A, Peng J, Liu Q et al. Efficient generation of functional dopaminergic neurons from human induced pluripotent stem cells under defined conditions. Stem Cells 28, 1893-1904 (2010).

4 Kroon E, Martinson LA, Kadoya K et al. Pancreatic endoderm derived from human embryonic stem cells generates glucose-responsive insulin-secreting cells in vivo. Nat. Biotechnol. 26, 443-452 (2008).

5 Pagliuca FW, Millman JR, Gurtler M et al. Generation of functional human pancreatic $\beta$ cells in vitro. Cell 159(2), 428-439 (2014).

6 Schwartz SC, Hubschman J-P, Heilwell G et al. Embryonic stem cell trials for macular degeneration: a preliminary report. Lancet 379, 713-720 (2012). 
7 Schwartz SD, Regillo CD, Lam BL et al. Human embryonic stem cell-derived retinal pigment epithelium in patients with age-related macular degeneration and Stargardt's macular dystrophy: follow-up of two open-label Phase I/II studies. Lancet 385, 509-516 (2015).

8 Trounson A, McDonald C. Stem cell therapies in clinical trials: progress and challenges. Cell Stem Cell 17, 11-22 (2015).

9 Takahashi K, Yamanaka S. Induction of pluripotent stem cells from mouse embryonic and adult fibroblast cultures by defined factors. Cell 126, 663-676 (2006). regenerative medicine. Regen. Med. 10(3), 287-304 (2015).

11 Gould DS, Auchincloss H. Direct and indirect recognition: the role of MHC antigens in graft rejection. Immunol. Today 20(2), 77-82 (1999).

12 Meier-Kriesche HU, Schold JD, Srinivas TR, Kaplan B. Lack of improvement in renal allograft survival despite a marked decrease in acute rejection ratesover the most recent era. Am. J. Transplant. 4(3), 378-383 (2004).

13 Lamb KE, Lodhi S, Meier-Kriesche HU. Long-term renal allograft survival in the United States: a critical reappraisal. Am. J. Transplant. 11(3), 450-462 (2011).

14 Drukker M, Katz G, Urbach A et al. Characterisation of the expression of MHC proteins in human embryonic stem cells. Proc. Natl Acad. Sci. USA 99, 9864-9869 (2002).

15 Li L, Baroja ML, Majumdar A et al. Human embryonic stem cells possess immune-privileged properties. Stem Cells 22(4), 448-456 (2004).

16 Imberti B, Monti M, Casiraghi F. Pluripotent stem cells and tolerance induction in organ transplantation. Curr. Opin. Organ Transplant. 20, 86-93 (2015).

17 Trigona WL, Porter CM, Horvath-Arcidiacono JA, Majumdar As, Bloom ET. Could heme-oxygenase-1 have a role in modulating the recipient immune response to embryonic stem cells? Antioxid. Redox Signal 9(6), 751-756 (2007).

18 Yachimovich-Cohen N, Even-Ram S, Shufaro Y, Rachmilewitz J, Reubinoff B. Human embryonic stem cells suppress $\mathrm{T}$ cell responses via arginase 1-dependent mechanism. J. Immunol. 184, 1300-1308 (2010).

19 Sugita S, Kamao H, Iwasaki Y et al. Inhibition of T-cell activation by retinal pigment epithelial cells derived from induced pluripotent stem cells. Invest. Ophthalmol. Vis. Sci. 56, 1051-1062 (2015).

20 Kim K, Doi A, Wen B et al. Epigenetic memory in induced pluripotent stem cells. Nature 467, 285-290 (2010).

21 Polo JM, Liu S, Figueroa ME et al. Cell type of origin influences the molecular and functional properties of mouse induced pluripotent stem cells. Nat. Biotechnol. 28(8), 848-855 (2010).

22 Nasr IW, Wang Y, Gao G et al. Testicular immune privilege promotes transplantation tolerance by altering the balance between memory and regulatory T cells. J. Immunol. 174, 6161-6168 (2005).

23 Wang X, Qin J, Zhao RC, Zenke M. Reduced immunogenicity of induced pluripotent stem cells derived from Sertoli cells. PLoS ONE 9, e106110 (2014).
24 Liu P, Chen S, Li X et al. Low immunogenicity of neural progenitor cells differentiated from induced pluripotent stem cells derived from less immunogenic somatic cells. PLoS ONE 8(7), e69617 (2013).

25 Drukker M, Katchman H, Katz G et al. Human ES cells and their differentiated derivatives are less susceptible to immune rejection than adult cells. Stem Cells 24, 221-229 (2006).

26 Swijnenburg R-J, Tanaka M, Vogel H et al. Embryonic stem cell immunogenicity increases upon differentiation after transplantation into ischemic myocardium. Circulation 112, I-166-I-172 (2005).

27 Nussbaum J, Minami E, Laflamme MA et al. Transplantation of undifferentiated murine embryonic stem cells in the heart: teratoma formation and immune response. FASEB J. 21, 1345-1357 (2007).

28 Kofidis T, de Bruin JL, Tanaka $\mathrm{M}$ et al. They are not stealthy in the heart: embryonic stem cells trigger cell infiltration, humoral and T-lymphocyte-based host immune response. Eur. J. Cardiothorac. Surg. 21, 461-466 (2005).

29 Fairchild PJ, Nolan KF, Cartland S, Waldmann H. Embryonic stem cells and the challenge of transplantation tolerance. Trends Immunol. 25, 465-470 (2004).

30 Fairchild PJ, Robertson NJ, Cartland S, Nolan KF, Waldmann H. Cell replacement therapy and the evasion of destructive immunity. Stem Cell Rev. 1(2), 159-167 (2005).

31 Robertson NJ, Brook F, Gardner RL, Cobbold SP, Waldmann H, Fairchild PJ. Embryonic stem cell-derived tissues are immunogenic but their innate immune privilege promotes the induction of tolerance. Proc. Natl Acad. Sci. USA 104, 20920-20925 (2007).

- This paper derived a panel of embryonic stem cell (ESC) lines representing escalating levels of immunogenicity in order to define the extent of disparity that might be tolerated in the context of cell replacement therapy without recourse to immune suppression.

32 Turner M, Leslie S, Martin NG et al. Towards the development of a global induced pluripotent stem cell library. Cell Stem Cell 13, 382-384 (2013).

33 Solomon S, Pitossi F, Rao MS. Banking on iPSC - is it doable and is it worthwhile? Stem Cell Rev. Rep. 11, 1-10 (2015).

34 Wilmut I, Leslie S, Martin NG et al. Development of a global network of induced pluripotent stem cell haplobanks. Regen. Med. 10(3), 235-238 (2015).

35 Taylor CJ, Bolton EM, Pocock S, Sharples LD, Pedersen RA, Bradley JA. Banking on human embryonic stem cells: estimating the number of donor cell lines needed for HLA matching. Lancet 366, 2019-2025 (2005).

- An important paper that first suggested the feasibility of constructing a bank of human ESC lines by calculating the number of lines required to cover a significant proportion of the UK population.

36 Lin G, Xie Y, OuYang Q et al. HLA-matching potential of an established human embryonic stem cell bank in China. Cell Stem Cell 5(5), 461-465 (2009).

37 Rao MS, Auerbach JM. Estimating human embryonic stem cell numbers. Lancet 367, 650 (2006). 
38 Nakatsuji N, Nakajima F, Tokunaga K. HLA-haplotype banking and iPS cells. Nat. Biotechnol. 26, 739-740 (2008).

39 Barry J, Hyllner J, Stacey G, Taylor CJ, Turner M. Setting up a haplobank: issues and solutions. Curr. Stem Cell Rep. 1(2), 110-117 (2015).

40 Fairchild PJ. The challenge of immunogenicity in the quest for induced pluripotency. Nat. Rev. Immunol. 10, 868-875 (2010).

41 Wu D, Boyd AS, Wood KJ. Embryonic stem cells and their differentiated derivatives have a fragile immune privilege but still represent novel targets of immune attack. Stem Cells 26(8), 1939-1950 (2008).

42 Chen T-C, Waldmann H, Fairchild PJ. Induction of dominant transplantation tolerance by an altered peptide ligand of the male antigen, Dby. J. Clin. Invest. 113, 1754-1762 (2004).

43 de Almeida PE, Meyer EH, Kooreman NG et al. Transplanted terminally differentiated induced pluripotent stem cells are accepted by immune mechanisms similar to self-tolerance. Nat. Commun. 5, 3903 (2014).

44 Qin S, Cobbold SP, Pope H et al. 'Infectious' transplantation tolerance. Science 259, 974-977 (1993).

45 Samstein RM, Josefowicz SZ, Arvey A, Treuting PM, Rudensky AY. Extrathymic generation of regulatory T cells in placental mammals mitigates maternal-fetal conflict. Cell 150, 29-38 (2012).

46 Rong Z, Wang M, Hu Z et al. An effective approach to prevent immune rejection of human ESC-derived allografts. Cell Stem Cell 14, 121-130 (2014).

47 Lui KO, Howie D, Ng S-W, Liu S, Chien KR, Waldmann $\mathrm{H}$. Tolerance induction to human stem cell transplants with extension to their differentiated progeny. Nat. Commun. 5 , 5629 (2014).

- A landmark paper demonstrating that the induction of transplantation tolerance to pluripotent stem cell extends to antigens subsequently expressed by their differentiated progeny.

48 Pearl JI, Lee AS, Leveson-Gower DB et al. Short-term immunosuppression promotes engraftment of embryonic and induced pluripotent stem cells. Cell Stem Cell 8, 309-317 (2011).

49 Szot GL, Yadav M, Lang J et al. Tolerance induction and reversal of diabetes in mice transplanted with human embryonic stem cell-derived pancreatic endoderm. Cell Stem Cell 16, 148-157 (2015).

50 Grinnemo KH, Genead R, Kumagai-Braesch M et al. Costimulation blockade induces tolerance to HESC transplanted to the testis and induces regulatory $\mathrm{T}$ cells to HESC transplanted to the heart. Stem Cells 26, 1850-1857 (2008).

51 Ljung K, Simonson OE, Felldin U et al. Costimulation blockade induces Foxp $3^{+}$regulatory T cells to human ES cells. BioResearch Open Access 2, 455-458 (2013).

52 Lui KO, Boyd AS, Cobbold SP, Waldmann H, Fairchild PJ. A role for regulatory $\mathrm{T}$ cells in acceptance of embryonic stem cell-derived tissues transplanted across an MHC barrier. Stem Cells 28, 1905-1914 (2010).
53 Pan Y, Leveson-Gower DB, de Almeida PE et al. Engraftment of embryonic stem cells and differentiated progeny by host conditioning with total lymphoid irradiation and regulatory T cells. Cell Rep. 10, 1793-1802 (2015).

- An important paper showing the indefinite survival of tissues differentiated from ESCs following the induction of tolerance using protocols amenable to clinical translation.

54 Yu J, Vodyanik MA, Smuga-Otto K et al. Induced pluripotent stem cell lines derived from human somatic cells. Science 318, 1917-1920 (2007).

55 Takahashi K, Tanabe K, Ohnuki M et al. Induction of pluripotent stem cells from adult human fibroblasts by defined factors. Cell 131, 861-872 (2007).

56 Reardon S, Cyranoski D. Japan stem cell trial stirs envy. Nature 513, 287-288 (2014).

57 Zhao T, Zhang Z-N, Rong Z, Xu Y. Immunogenicity of induced pluripotent stem cells. Nature 474, 212-215 (2011).

- The first paper to describe immunogenicity associated with syngeneic-induced pluripotent stem cells, raising important questions about the impact of developmental antigens on the rejection of tissues derived from autologous cell lines.

58 Zhao T, Zhang Z-N, Westenskow PD et al. Humanized mice reveal differential immunogenicity of cells derived from autologous induced pluripotent stem cells. Cell Stem Cell 17, 353-359 (2015).

59 Pangas SA, Yan W, Matzuk MM, Rajkovic A. Restricted germ cell expression of a gene encoding a novel mammalian HORMA domain-containing protein. Gene Exp. Patterns 5 , 257-263 (2004).

60 Daniel K, Lange J, Hached K et al. Meiotic homologue alignment and its quality surveillance are controlled by mouse HORMAD1. Nat. Cell Biol. 13, 599-610 (2011)

61 Chen Y-T, Venditti CA, Theiler G et al. Identification of CT46/HORMAD1, an immunogenic cancer/testis antigen encoding a putative meiosis related protein. Cancer Immunity 5, 1-8 (2005).

62 Shahzad MMK, Shin Y-H, Matsuo K et al. Biological significane of HORMA domain containing protein 1 (HORMAD1) in epithelial ovarian carcinoma. Cancer Letters 330, 123-129 (2013).

63 Maynard KM, Arvindam U, Cross M, Firpo MT. Potentially immunogenic proteins expressed similarly in human ESC and iPSC. Exp. Biol. Med. 239, 484-488 (2014).

64 Araki R, Uda M, Hoki Y et al. Negligible immunogenicity of terminally differentiated cells derived from induced pluripotent or embryonic stem cells. Nature 494, 100-104 (2013).

65 Wang L, Cao J, Wang Y et al. Immunogenicity and functional evaluation of iPSC-derived organs for transplantation. Cell Discovery 1, 15015 (2015).

66 Lu Q, Yu M, Shen C et al. Negligible immunogenicity of induced pluripotent stem cells derived from human skin fibroblasts. PLoS ONE 9(12), e114949 (2014).

67 Guha P, Morgan JW, Mostoslavsky G, Rodrigues NP, Boyd AS. Lack of response to differentiated cells derived from syngeneic iPS cells. Cell Stem Cell 12, 407-412 (2013). 
Kamao H, Mandai M, Okamoto S et al. Characterization of human induced pluripotent stem cell-derived retinal pigment epithelium cell sheets aiming for clinical application. Stem Cell Rep. 2, 205-218 (2014).

69 Morizane A, Doi D, Kikuchi T et al. Direct comparison of autologous and allogeneic transplantation of iPSC-derived neural cells in the brain of nonhuman primates. Stem Cell Rep. 1, 1-10 (2013).
70 Ben-David U, Benvenisty N. The tumorigenicity of human embryonic and induced pluripotent stem cells. Nat. Rev. Cancer 11, 268-277 (2011).

71 Lee AS, Tang C, Rao MS, Weissman IL, Wu JC.

Tumorigenicity as a clinical hurdle for pluripotent stem cell therapies. Nat. Med. 19(8), 998-1004 (2013). 